\title{
STRATIFICATIONS PARFAITES ET THÉORIE DES POIDS
}

\author{
VICEN'TE NAVARRO AZNAR
}

\begin{abstract}
In this pirper, we emphasize Deligne's theory of weights, in order to prove that some stratificalions of algebraic varietics are perfect. In particular, we study in some detail the Bialynicki-Birula's stratifications and the stratifications considered by $\mathbf{F}$. Kirwan to compute the cohomology of syrriplectic or geometric quotients. Finaliy we also appoint the motivic formulation of this approsch, which contains the Hodge theoretic formulation.
\end{abstract}

\section{Introduction}

Il est bien conmu que l'espace projectif complexe $P^{n}(C)$ admet une filtration $\mathbb{P}^{n}(\mathbb{C}) \supseteq \mathbb{P}^{n-1}(\mathbb{C}) \supseteq \cdots$ qui donne une décomposition cellulaire $\mathbb{P}^{n}(\mathbb{C})=\mathbb{C}^{n} \cup \mathbb{C}^{n-1} \cup \ldots$ à partir de laquelle on peut obtenir les nombres de Betti du $\mathbb{P}^{n}(\mathbb{C})$ familiers à tous. Fin outre, on peut changer légèrement le point de vue et obtcrir cette décomposition cellulaire du $\mathbb{P}^{n}(\mathbb{C})$ de la théorie de Morse, car $\mathbb{P}^{n}(\mathbb{C})$ admet ine fonction de Morse avec $n+1$ points criliques d'index $0,2, \ldots, 2 n$, et il résulte alors de la parité de ces index que les inégalités générales de Morse entre nombres de Betti et nombres de points critiques sont daus ce cas des égalités. On peut finalenent considérer la réduction de $\mathrm{P}^{n}(C)$ à un corps fini $F_{q}$ : on obtient alors la décomposition $\mathbb{P}^{n}\left(\mathbb{F}_{q}\right)=\mathbb{F}_{q}^{n} \cup \mathbb{F}_{q}^{r-1} \cup \cdots$ qui permet de compter les points de $\mathbb{P}^{n z}\left(\mathbb{F}_{q}\right)$, obtenir la fonction zeta de $\mathbb{P}^{n}\left(\boldsymbol{F}_{q}\right)$ et retrouver, de nouveau, les nombres de Betti par les conjectures de Weil.

Dans cet article, nous nous intéressons aux décompositions ou stratifications des variêtés algébriques et aux fonctions de Morse sur celles-ci provenant de l'action d'un grouje algébrique sur la variété. Dans ce cas, la théorie dess poids, qui est néé des conjectures de Weil, nous permet de

Co travail a été partiellement subventionné par le project CICYT PS 90-0069. 
formuler un principe qui établit que toutes ces décompositions et fonctions de Morse sont parfaites, c'est-à-dire que pour elles les inćgalités de Morse sont toujours des égalités. Nous préciserons ce principe pour les décompositions de Bialynicki-Birula des variétés complètes lisses sur lesquelles opère un tore algébrique et pour les fonctions de Morse qui apparaissent comme carré de la norme de l'application moment de l'action d'un groupe algébrique réductif qui opère linéairement sur une variété projective complexe.

Remarquons que Atiyah-Bott ont donné dans [1] un critère pour qu'une stratification soil parfaite en termes de cohomologie équivariante et que, en utilisarnt ce critère, Kirwan $([15],[16])$ a déjà obtenu la plupart des résultats de cette note, bien qu'uniquement pour les décompositions filtrables, car le critère de Atiyah-Bott n'est disponible que pour ces décompositions. Par contre, l'approche de cette note, basé sur la théorie des poids, n'a pas besoin de cette hypothèse, ce qui infirme l'opinion de Carrell et Sommese, pour qui cette hypothcse paraissait essentielle ([6]).

\section{Poids et caractéristiques d'Euler pures}

Dans cette note, $k$ désigne un corps algébriquement clos de caractéristique $p \geq 0$, et on appelle $k$-schérnas les schémas séparés et de type fini sur $k$.

On fixe un premier $l$ distinct de $p$, et pour tout $k$-schéma $X$, on note $H^{*}(X)$, resp. $H_{c}^{*}(X)$, la cohomologie $l$-adique, resp. à support compact, de $X$, c'est-à-dire qu'or pose

$$
H^{*}(X)=H^{*}\left(X, \mathbb{Q}_{i}\right)
$$

et

$$
H_{c}^{*}(X)=H_{c}^{*}\left(X, \mathbb{Q}_{l}\right)
$$

Si $p=0$ et $k$ est un sous-corps de $\mathbb{C}$, on peut alternativernent considérer la cohomologie singulière rationnelle au lieu de la cohomologie $l$-adique, c'est-è-dire que dans ce cas le lecteur pourra interpréter dans tout ce qui suit $H^{*}(X)$ et $H_{c}^{*}(X)$, par $H^{*}(X \otimes \mathbb{C}, Q)$ et $H_{c}^{*}(X \otimes \mathbb{C}, \mathbb{Q})$, respectivement.

D'après la théorie des poids de Deligne ([7]), on sait que ces groupes de cohomologie ont une filtration croissante: la filtration par le poids $W$, et à partir de celle-ci or peut introduire les caractéristiques d'Fuler pures de $X$, définies, suivant Durfee ([11], mais où elles sont appelées mixtes), par

$$
\chi_{m}(X)=\sum_{i}(-1)^{i} \operatorname{dim} G r_{m}^{W} H^{i}(X)
$$


et

$$
\chi_{c}^{m}(X)=\sum_{i}(-1)^{i} \operatorname{dim} \operatorname{Cr}_{m n}^{w} H_{c}^{i}(X) .
$$

En particulier, si la structure de $H^{i}(X)$ est, pure de poids $i$, i.e.

$$
G r_{m}^{W} H^{i}(X)=0, \text { pour tout } m \neq i
$$

ce qui est, par exemple, le cas si $X$ est lisse et complet, et on note $b_{i}(X)$ les nombres de Betti de $X$, on a

$$
\chi_{2}(X)=b_{i}(X)
$$

Mais, en général, ils difèrent et ces caractéristiques d'Euler pures ont un meilleur comportement que les nombres de Betti face aux suites exactes de cohomologie.

Finalement, nous introduisons les polynômes de Poincaré purs de $X$ par

$$
P_{p u r}(X, t)=\sum_{m} \chi_{m}(X) t^{m}
$$

et

$$
P_{p_{t u}}^{c}(X, t)=\sum_{m} \chi_{i}^{n t}(X) t^{m}
$$

\section{Décompositions et stratifications}

Puisque les définitions de décomposition ct stratification d'une variété algćbrique varient parfois suivant les auteurs, nous allons préciser notre terminologic.

Définitions. Soit $X$ un $k$-schéma, tue décomposition de $X$ est une famille finie de sous-schérnas localement fermés de $X:\left\{X_{\alpha}\right\}_{\alpha \in A}$, qui sont disjoints et tels que $X=\cup X_{\gamma}$. On appelle cellules de ha décomposition les sous-schémas $X_{t x}$.

Une décorriposition de $X$ est dite filtrable s'il existe une suite décroissante $F_{1} \supseteq F_{2} \supseteq \cdots \supseteq F_{m+1}$ de sous-schémas fermés de $X$ telle que $F_{1}=X, F_{n+1}=\phi$ et $F_{\beta}-F_{\beta+1}$ est une cellule de la décomposition, $\beta=1, \ldots, m$.

Une stratification de $X$ est une décomposition telle que la frontière de toute cellule est une réunion de cellules.

Il est clair que toute stratification est une décomposition filtrable, et qu'il y a des décompositions filtrables qui ne sont pas des stratifications. 
Théorème 1 (cf. [11]). Soit $X$ un $k$-schéma et soit $X=\cup X_{\alpha}$ une décomposition de $X$. Alors on a

$$
\chi_{c}^{m}(X)=\sum_{\alpha} \chi_{c}^{m}\left(X_{\alpha}\right), \quad \text { pour tout } m
$$

Démonstration: Si $N=\operatorname{dim} X$ et $X_{1}, X_{2}, \ldots, X_{r}$ sont les cellules de $X$ de dimension $N$, on va prouver le résultat par double récurrence sur $N$ et $r$. Le cas $N=0$ étant évident, on suppose $N>0$ et $r \geq 1$.

Si une des cellules $X_{\beta}, \beta=1, \ldots, r$, est un ouvert de $X$, par récurrence; il suffit de prouver le cas particulier suivant du théorème: si $X=X_{1} \cup X_{2}$ et $X_{1}$ est un olvert de $X$, on a

$$
\chi_{c}^{m}(X)=\chi_{c}^{m}\left(X_{1}\right)+\chi_{c}^{m n}\left(X_{2}\right) .
$$

En effet, ceci résulte immédiatement de la suite exacte

$$
\cdots \rightarrow H_{c}^{i}\left(X_{1}\right) \rightarrow H_{c}^{i}(X) \rightarrow H_{c}^{i}\left(X_{2}\right) \rightarrow \cdots
$$

car le foncteur $\mathrm{Cr}_{m}^{W}$ est exact.

Dans le cas général, soit $\stackrel{\circ}{X}_{r}$ l'intérieur de $X_{\tau}$. Il est clair que la décomposition

$$
X-\stackrel{\circ}{X}_{r}=\bigcup_{a \neq r} X_{\alpha} \cup\left(X_{r}-\stackrel{\circ}{X}_{r}\right)
$$

a $r-1$ cellules de dimension $N$, donc par récurrence on a

$$
\chi_{c}^{m}\left(X-\stackrel{\circ}{X}_{r}\right)=\sum_{\alpha \neq r} \chi_{c}^{m}\left(X_{\alpha}\right)+\chi_{e}^{m}\left(X_{r}-\stackrel{\circ}{X}_{r}\right) .
$$

Puisque pour les décompositions

$$
X=\stackrel{\circ}{X}_{r} \cup\left(X-\stackrel{\circ}{X}_{r}\right)
$$

et

$$
X_{r^{*}}=\stackrel{\circ}{X}_{r} \cup\left(X_{r}-\stackrel{\circ}{X}_{r}\right)
$$

on peut appliquer le cas particulier du théorème déjà prouvé, on obtient

$$
\chi_{c}^{m}(X)=\chi_{c}^{m}\left(\stackrel{\circ}{X}_{r}\right)+\chi_{c}^{m}\left(X-\stackrel{\circ}{X}_{r}\right)
$$

et

$$
\chi_{c}^{m}\left(X_{r}\right)=\chi_{c}^{m}\left(\stackrel{\circ}{X}_{r}\right)+\chi_{c}^{m}\left(X_{r}-\stackrel{\circ}{X}_{r}\right)
$$

d'oú il résulte le théorème. 
Corollaire. Si $X$ est lisse et connexe, et les cellules $X_{\alpha}$ sont lisses, équidimensionnelles et de codimension $d_{\alpha}$ dans $X$, on a

$$
\chi_{m}(X)=\sum_{0} \chi_{m-2 t_{o}}\left(X_{\alpha}\right)
$$

Dérnonstration: En effet, par dualité de Poincaré, on a

$$
\chi_{c}^{m}(X)=\chi_{2 \operatorname{sim} X-m}(X)
$$

et

$$
\chi_{c}^{m}\left(X_{\alpha}\right)=\chi_{2 \operatorname{dim}} X_{c}-m\left(X_{\sigma}\right)
$$

donc le résultat suit immédiatement du théorène 1 .

\section{Décompositions et stratifications parfaites}

Soit $X=\left\llcorner X_{a}\right.$ une stratification (ou même une décomposition filtrable) de $X$; il est clair, en changeant les sons-index si nécessaire, qu'il existe une filtration de $X$ par des sous-schémas fermés

$$
F_{1} \supseteq F_{2} \supseteq F_{3} \supseteq \cdots
$$

telle que

$$
F_{\alpha}-F_{\alpha+1}=X_{\alpha}
$$

pour tout $\alpha$. Alors, pour tout $\alpha$, on a une suite exacte

$$
\cdots \rightarrow H_{c}^{i}\left(X_{\alpha}\right) \rightarrow H_{c}^{i}\left(F_{\alpha}\right) \rightarrow H_{c}^{i}\left(F_{\alpha x+1}\right) \rightarrow \cdots
$$

et on dit que la stratification est parfaite si tontes ces suites se scindent en des suites exactes courtes

$$
0 \rightarrow H_{c}^{i}\left(X_{\alpha}\right) \rightarrow H_{c}^{i}\left(F_{\alpha}\right) \rightarrow H_{c}^{i}\left(F_{\alpha+1}\right) \rightarrow 0
$$

Si $X$ est lisse et connexe, toutes les cellules sont lisses et connexes, et notons $d_{\alpha}$ la codimension de $X_{\alpha}$ dans $X$, il en résulte alors que

$$
b_{i}(X)=\sum_{a} b_{i-2 d_{\alpha}}\left(X_{\alpha}\right), \text { pour tout } i .
$$

En général, si $X$ es lisse et connexe et $X=\cup X_{n}$ est une décomposition de $X$, avec des cellules lisses et connexes, nous dirons qu'elle est parfaite si elle vérifie l'égalité antérieure entre nombres dc Betti. 
Nous nous intéressons plus particulièrement aux décompositions de Bialynicki-Birula ([2]), qui, en gćnéral, ne sont filtrables que dans le cas projectif ([4], [14]). Rappelons que ces décompositions s'obtiennent de la manière suivante.

Soit $X$ un $k$-schéma lisse et complet sur lequel agit un tore algébrique $T$ el soit $X^{T}=\cup X_{\alpha}^{T}$ la décomposition de la partie fixe $X^{T}$ en composantes connexes. Alors $X$ a une décomposition $X=\cup X_{\alpha}$, telle que $X_{\alpha}$ est, un $k$-schéma lisse, $X_{\alpha}^{\gamma}$ est un sous-schéma fermé de $X$ et $X_{\alpha}$ est une fibration sur $X_{\alpha}^{T}$, en particulier, le morphisme

$$
H^{*}\left(X_{\alpha}\right) \rightarrow H^{*}\left(X_{\alpha}^{T}\right)
$$

est un isomorphisme.

Ces décompositions sont le sujet du résultat suivant.

Théorème 2. Soit $X$ un k-schéma connexe, lisse et complet, qui admet une decomposition $X=\cup X_{\alpha}$, telle que chaque $X_{\alpha}$ est un $k$-schéma lisse, connexe, de codimension $d_{\alpha}$ et a un sous-schéma complel $Y_{\alpha}$ pout lequel le morphisme

$$
H^{*}\left(X_{\alpha}\right) \rightarrow H^{*}\left(Y_{\alpha}\right)
$$

est un isomorphisme. Alors,

$$
b_{i}(X)=\sum_{\alpha} b_{i-2 d_{\alpha}}\left(Y_{\alpha}\right), \text { pour tout } i,
$$

et, donc, la décomposition de $X$ est parfaite.

Démonstration: Puisquc aussi bien $X$ que les cellules $X_{a}$ sont lisses, et puisqu'on a

$$
\chi_{m}\left(X_{\alpha}\right)=\chi_{m n}\left(Y_{\alpha}\right)
$$

il résulte du corollaire au théorème 1 que

$$
\chi_{m}(X)=\sum_{\alpha} \chi_{m-2 d_{\alpha}}\left(Y_{\alpha}\right)
$$

Or on a

$$
G r_{m}^{W} H^{i}\left(X_{\alpha}\right)=0, \text { si } m<i,
$$

car les $X_{\alpha}$ sont lisses, et on a

$$
G r_{m}^{W} H^{i}\left(Y_{\alpha}\right)=0, \text { si } m>i
$$


car les $Y$ sont complets, d'où

$$
G r_{m}^{W} H^{i}\left(Y_{o}\right)=0, \quad \text { si } m \neq i
$$

et il en résulte que

$$
\chi_{m}\left(Y_{c}\right)=b_{m}\left(Y_{\alpha}\right)
$$

Puisqu'on a aussi

$$
\chi_{m}(X)=b_{m}(X)
$$

$X$ étant lisse et complet, ceci prouve le théorème.

Corollaire (cf. [3], [6], [12], [16]). Soit $X$ ur $k$-schéma connexe, lisse et conplet sur lerpat agit un tore algébrique T. Si $X^{T}=\cup X_{\alpha}^{T}$ est la décomposition de $X^{T}$ en composantes connexes el $d_{\alpha}$ est la codimension. de la cellute $X_{n}$ de la décomposition de Bialynichi-Birula de $X$, on a

$$
b_{\imath}(X)=\sum_{\alpha} b_{i-2 t_{n}}\left(X_{\alpha}^{T}\right), \text { pour toul } \quad i .
$$

Remarque. Daus [3] les nombres de Betti de $X$ sont donnés par une expresion équivalente à l'antérieure mais légèromont différente. En effet. rappelons que, associées à la décomposition en composantes comexes de $X^{T}$; on a deux décompositions de $X$

$$
X=\bigcup_{\sigma \in A} X_{\alpha}^{+}=\bigcup_{a \in A} X_{\alpha r}^{-} .
$$

Alors, la formule du corollaire antéricur pour la décomposition $X=$ $\cup X_{c}^{+}$donne

$$
b_{i}(X)=\sum b_{i-2 d_{a}}\left(X_{c r}^{T}\right)
$$

ou $d_{a}=\operatorname{dim} X-\operatorname{dim} X_{a}^{+}$. Tandis que la formule de [3]: pour cette décomposition, est

$$
b_{i}(X)=\sum b_{i-2 t_{t s}^{+}}\left(X_{a}^{T}\right)
$$

où $d_{\alpha}^{+}=\operatorname{dim} X_{\alpha}^{+}-\operatorname{dim} X_{\alpha}^{\gamma}$. Or, puisqu'on a

$$
\operatorname{dim} X=\operatorname{dim} X_{a}^{+}+\operatorname{dim} X_{t, 2}^{-}-\operatorname{dim} X_{a}^{T}
$$

il résulte que

$$
d_{n}=\operatorname{dim} X_{a x}^{-}-\operatorname{dim} X_{c x}^{\tau}\left(=: d_{c}^{-}\right)
$$

et ainsi la formule du corollaire olbtenue à partir de la décornposition $X=$ $\cup X_{\alpha}^{+}$coincide avec la formule de [3] obtenue à partir de la décomposition $X=\cup X_{\alpha}^{-}$. 


\section{Théorie équivariante}

Soit $G$ un groupe $k$-algébrique linéaire et $X$ un $G$-schéma, i.e. un $k$ schema sur lequel $G$ agit, la cohomologie $l$-adique équivariante de $X$ est définie comme la cohomologie $l$-adique du schéma sinplicial $B(X, G)$, obtenu par la construction barre géométrique; ainsi: si on suppose, par exemple, que $G$ agit à droite sur $X$, on a

$$
B(X, G)_{n_{k}}=X \times G^{n}
$$

et

$$
\begin{aligned}
& \delta_{0}\left(x, g_{1}, g_{2}, \ldots, g_{n}\right)=\left(x g_{1}, g_{2}, \ldots, g_{n}\right) \\
& \delta_{i}\left(x, g_{1}, g_{2}, \ldots, g_{n}\right)=\left(x, g_{1}, \ldots, g_{i} g_{i+1}, \ldots, g_{n}\right),
\end{aligned}
$$

si $1 \leq i \leq n-1$, et

$$
\delta_{n}\left(x, g_{1}, g_{2}, \ldots, g_{n}\right)=\left(x, g_{1}, \ldots, g_{n-1}\right),
$$

(voir $[8,6.1]$ ). Il en résulte que les groupes de cohomologie équivariante $H_{G}^{*}(X)$ et $H_{G, c}^{*}(X)$ ont aussi une filtration par le poids, qui vérifie le théorème suivant ( $\mathrm{cf} .[8,(8.2 .4)]$ ):

Théorème 3. Soit $X$ un $G$-schéma de dimension $N$. Pour tout $i \geq 0$, on a:

1) Les entiers $m$ lels que $G r_{m}^{W} H_{G}^{i}(X)$ est non nul vérifient les conditions suivantes:

(i) $m \in[0,2 i] \cap[i-N, i+N]$.

(ii) Si $X$ est lisse, $m \in[i, 2 i] \cap[i, i+N]$.

(iii) Si $X$ est complet, $m \in[0, i] \cap[i-N, i]$.

2) Les entiers $m$ tels que $G r_{m}^{W} H_{G, c}^{i}(X)$ est non nul vérifient $m \in$ $[0, i] \cap[i-N, i]$.

Démonstration: Les différentes parties du théorèrre résultent des propriétés des poids des faiscealux d'irnages directes $\mathbb{R}^{i} f_{*} \mathbb{Q}_{l}$ du morphisme

$$
f: B(X, G) \rightarrow B(G)
$$

analogue simplicial du morphisme classique

$$
X \times U_{G} / G \rightarrow B_{G}
$$


car $B(G)$ se comporte cohomologiquenent conme un schéma lisse et complet, c'est-à-dire, que si $F$ cst un faisceau lisse pur de poids $n$ sur $B(G)$, alors $H^{i}(B(G), F)$ est pur de poids $n+i$ (cf. $[9,(3.3 .6)]$ et $[8,(9.1 .4)])$. Nous nous bornons à domer une esquisse de preuve du théorène.

Ein général, le faisceau $\mathbb{R}^{i} f_{*} \mathbb{Q}_{l}$ est mixte de poids dans $[0,2 i] \cap[i-$ $N, i+N]([9,(3.3 .8)])$, donc on obtient I) (i).

Pour prouver 2), on considère les faiscenux d'images directes à support propre $R^{i} f !$ Q qui sont mixtes de poids clans $[0, i] \cap[i-N, i]([9,(3.3 .8)])$; et ainsi, les groupes $H_{C, c}^{i}(X)$ sont mixtes de poids aussi dans $[0, i] \cap[i-$ $N, i]$.

Si $X$ est lisse de dimension $N$, le faisceat $\mathbb{R}^{i} f_{*} Q_{l}$ est le dual du faisceau $\mathbb{R}^{2 N-i} f_{1} Q_{t}(N)$, est donc mixte de poids dans $[i, 2 i] \cap[i, i+N]$, d'oú il résulte 1) (ii).

Evidemınent, 1) (iii) est un cas particulier de 2).

Si on dispose dé la résolution équivariarte de singularités, par exemple si $h$ est de caractéristique 0, on pent clonner une alutre preuve de ce théorme, mêne en termes de structures de Hodge mixtes: car on dispose alors des ingrédierts essentiels pour suive la ligne de démonstration de Deligne darns le cas non équivariant ([8]). En ellet, si $X$ est lisse et complet on a

$$
H_{G}^{*}(X)=\left(H^{*}(X) \otimes H^{*}\left(B_{G}\right)\right)^{G / G_{01}}
$$

où $G_{0}$ est la composante connexe de $G$ (voir $[15,5.8]$ ), donc ces groupes ont des structures pures. Si $X$ est lisse et non complet, par Sumihiro $[17]$ et Hironaka, il existe un $G$-schéma lisse et complet $\bar{X}$ qui contient $X$ comme un owvert de Zariski dense et tel que $\bar{X}-X$ est un diviseur à croisements normaux, or peut alors suivre les argurnents de $[8]$ et pronver 1) (ii). Si $X$ est propre, par Hironaka, il existe une byperrésolution équivariante de $X$ et il en résulte 1) (iii) par descente cohomologique. D'oú on déduil aussi 2) en utilisant encore le théorèrne de complétation équivariante et la suite exacte de cohomologie correspondante. Finalement, il en résulte aussi 1) (ii) par descente colomologique.

Il résulte de ce théorème que pour la cohomologie équivariante les caractéristiques d'Euler pures sont définics, bicn que la caractéristique d'Euler ordinaire n'est pas en général définic, car on peut avoir un nombre infini de groupes de cohornologie non ruuls. On définit donc les caractéristiques d'Euler équivariantes pures de $X$ par

$$
\chi_{C}^{T h}(X)=\sum_{i}(-1)^{i} \operatorname{dim} G r r_{m}^{W} H_{G}^{i}(X)
$$


et

$$
\chi_{G, c}^{m}(X)=\sum_{i}(-1)^{i} \operatorname{dim} G r_{m}^{W} H_{G, c}^{i}(X) .
$$

On définit la série de Poincaré équivariante pure de $X$ par

$$
P_{p u T}^{G}(X)=\sum_{m} \chi_{C}^{m}(X) t^{m}
$$

qui, éventuellement, peut être une série formelle non polynồniale.

Si $X$ est un $G$-schéma, on introduit, comme dans le paragraphe 2, les $G$-décompositions, les $G$-décompositions filtrables el les $G$-stratifications, et une démonstration parallèle à celle du théorème 2 donne le résultat suivant.

Proposition 4. Soit $X$ un $G$-schéma et soit $X=\cup X_{\alpha}$ un $G$-décomposition de $X$. Alors on a

$$
\chi_{G, c}^{m}(X)=\sum_{\alpha} \chi_{G, c}^{m}\left(X_{\alpha}\right), \text { pour tout } m
$$

La dualité de Poincaré n'étant pas valable en cohomologie équivariante, pour obtenir l'analogue équivariant du corollaire au théorèrne 1 , nous devons utiliser la cohomologie locale équivariante et introduire les caractéristiques d'Eulcr pures correspondantes.

Soient $X$ un $G$-schéma et $Y$ un $G$-sous-schéma localcment fermé de $X$. Alors, il existe un $G$-sous-schérna ouvert $U$ de $X$ tel que $Y$ est un fermé de $U, U-Y$ est donc aussi un $G$-schéma et on définit, la cohomologie locale à support dans $Y: H_{G, Y}^{*}(X)$, comme la cohomologie du cône du morphisine

$$
B(U-Y, G) \rightarrow B(U, G),
$$

(voir $[8,(6.3)]$ ), cohomologie qui ne dépend pas du ouvert $U$ choisi. Cette cohomologie a quelques propriétés en commun avec la cohomologie locale ordinaire, ainsi cette cohomologie a une filtration par le poids, vérifie la propriété d'excision, l'isomorphisme de Thon et, on a la suite exacte longue de cohomologie que, par exemple si $Y$ est fermé dans $X$, cst

$$
\cdots \rightarrow H_{G, Y}^{i}(X) \rightarrow H_{C}^{i}(X) \rightarrow H_{G}^{i}(X-Y) \rightarrow \cdots
$$

Proposition 5. Soient $X$ un $G$-schéma et $Y$ un $G$-souss-schéma tocalement fermé de $X$. Les entiers m tels que $G r_{m}^{W} H_{G, Y}^{i}(X)$ est non mul 
sont positifs et pour chague $m$ il n'y a qu'un nombre fini d'entiers $i \geq 0$ tels que $\mathrm{Gr}_{n}^{W} H_{C, Y}^{i}(X)$ est non nul.

Démonstration: Elle suit immédiatement de la suite exacte antériêre et du théorème 3 .

On peut donc défnir les caractéristiques d'Tuler pures pour cette cohomologie locale éguivariante par

$$
\chi_{G, Y}^{\eta n}(X)=\sum_{i}(-1)^{2} \operatorname{dim} G r_{m}^{W} H_{G, Y}^{i}(X) .
$$

Proposition 6. Soit $X$ un $G$-schéma et soit $X=\cup X_{\alpha}$ une $G-$ décomposition de $X$. Alors on a

$$
\chi_{G}^{m}(X)=\sum_{a} \chi_{G: X_{\mathrm{a}}}^{m}(X), \text { pour tout } m
$$

Démonstration: Il nous convient pour le raisonnement qui suit de prouver le résultat plus général suivant.

Soient $Z$ un $G$-schéma, $X$ un $G$-sous-schóma localement fermé de $Z$ et $X=\cup X_{a}$ une $G$-décomposition de $X$. Alors on a

$$
\chi_{G, X}^{m_{1}}(Z)=\sum_{G} \chi_{C, X_{\alpha}}^{m_{2}}(Z), \text { pour tout } m
$$

En cffet, nous pouvons maintenant suivre le même argument que dans la prêve du théorène 1 . Par la même récurrence, il nous suffit de prouver le cas où $X=X_{1} \cup X_{2}$ et $X_{1}$ est un ouvert de $X$. Mais dans ce cas, on a la suite exacte

$$
\cdots \rightarrow H_{C, X_{2}}^{i}(Z) \rightarrow H_{C, X}^{i}(Z) \rightarrow H_{G, X_{1}}^{i}(Z) \rightarrow \cdots,
$$

et on obtient

$$
\chi_{G, X}^{m i}(Z)=\chi_{G, X_{1}}^{m n}(Z)+\chi_{G_{1}, X_{2}}^{m}(Z) .
$$

Corollaire. Si $X$ est lisse el connexe et les cellules $X_{0}$ sont lisses, équidimensionnelles el de codimension $d_{\text {o }}$ dans $X$, on a

$$
\chi_{G}^{m}(X)=\sum_{a} \chi_{G}^{m-2 d_{\alpha}}\left(X_{\kappa}\right)
$$

et:

$$
P_{r u r}^{G i}(X)=\sum_{c} t^{2 d_{\omega}} P_{p u r}^{C ;}\left(X_{\alpha}\right)
$$


Démonstration: En effet, il résulte de l'isonorphisme de 'Thom

$$
H_{G}^{i}\left(X_{\alpha}\right) \stackrel{\sim}{\rightarrow} H_{G_{1}, X_{\alpha}}^{i+2 d_{\alpha}}(X)\left(d_{\alpha}\right)
$$

que

$$
\chi_{C}^{m}\left(X_{\sigma}\right)=\chi_{G ; X_{\circ}}^{m+2 d_{\alpha}}(X),
$$

donc le résultat suit de la proposition antéricure.

Exemple 1. Nous allons maintenant montrer comment on peut déduire du corollaire précédent les formules de F. Kirwan ([15]) qui donnent inductivement les nombres de Betti d'un quotient symplectique ou géométrique d'une variété. Nous rappelons, d'abord, le théorème de décomposition de [15], suivant l'approche algébrique de [15, Part II], et renvoyons à loc. cit, pour plus de détails ainsi que pour la relation avec la théorie de Morse et le carré de la norme de l'application moment en géométrie symplectique.

Soient $X$ un $k$-schéma projectif et lisse et $G$ un $k$-groupe algébrique réductif qui agit linéairement sur $X$. Alors, d'après [15], il existe une décomposition filtrable de $X$

$$
X=\bigcup_{\beta \in B} S_{\beta}
$$

telle que $S_{0}$ est l'ouvert, $X^{s s}$ des points serni-stables de $X$, et pour tout $\beta \in B, \beta \neq 0, S_{\beta}$ est isomorphe à $G \underset{P_{\beta}}{\times} Y_{\hat{\beta}}^{s s}$, où $Y_{\beta}^{s s}$ est un sous-schéma de $X$ localement fermé et lisse, et $P_{\beta}$ est ur sous-groupe parabolique de $G$. En plus, il existe une fibration algébrique localement triviale et $P_{\beta}$-équivariante

$$
p_{\beta}: Y_{\beta}^{s s} \rightarrow Z_{\beta}^{s s}
$$

avec des fibres des espaces affines, où $Z_{\beta}^{s s}$ est l'ouvert des points semistables, par rapport à un sous-groupe réductif maximal stab $\beta$ de $P_{\beta}$, d'un sous-schéma fermé et lisse $Z_{\beta}$ de $X$.

Soient $S_{\beta, m}$ les composantes connexes de $S_{\beta}$ de codinension $d(\beta, m)$. Alors, il résulte du corollaire précódent que

$$
\chi_{G}^{p}(X)=\sum \chi_{G}^{p-2 d(\beta, m)}\left(S_{\beta, m}\right), \text { pour tout } p
$$

ou bien, en termes des polynômes de Poincaré purs

$$
P_{p u r}^{G}(X)=\sum t^{2 d\left(\beta, m_{2}\right)} P_{p u r}^{G}\left(S_{\beta, m}\right)
$$


Soient $Y_{\beta, m}^{s s}$ et $Z_{\beta, m}^{s s}$ les composantes correspondantes de $Y_{\beta}^{s s}$ et $Z_{\beta}^{s s}$. Il résulte des propriétés de la cohomologie équivariarite que

$$
\begin{aligned}
& H_{G}^{*}\left(S_{\beta_{1}, m}\right) \cong H_{P_{\beta}}^{*}\left(Y_{\beta_{i} ; n}^{s s}\right), \\
& H_{P_{\beta}}^{*}\left(Y_{\beta, m}^{s s}\right) \cong H_{P_{\beta}}^{*}\left(Z_{\beta, n}^{s s}\right) \text {, }
\end{aligned}
$$

et

$$
H_{P_{\beta}}^{*}\left(Z_{\beta, m}^{s s}\right) \cong H_{s t a b \beta}^{*}\left(Z_{\beta, m}^{s s}\right)
$$

d'où

$$
P_{p u r}^{G}\left(S_{\beta, m}\right)=P_{p u r}^{s t a b \beta}\left(Z_{\beta, m}^{s s}\right)
$$

et on obtient la formule récursive

$$
P_{m a r}^{G}\left(X^{s s}\right)=P_{m e r}^{G}(X)-\sum_{\substack{\beta \in B \\ 0 \leq m<\operatorname{dim} X}} t^{2 d(\beta, m)} P_{p m r}^{s t a b \beta}\left(Z_{\beta, m}^{s s}\right)
$$

En utilisant pour chaque $Z_{\beta, n}^{s s}$ cette formule, on obtient récursivement des sous-schémas fermés et lisses $Z_{\underline{\beta}: m}$, où $\underline{\beta}=\left(\beta_{1}, \beta_{2}, \ldots, \beta_{q}\right)$ (voir [15, $16.4]$ ), telles que

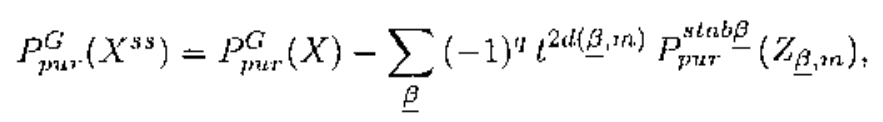

d'où, si $G$ est connexe, il résulte

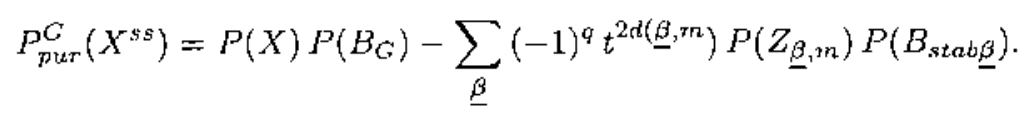

Finalement, si on suppose $X^{s}=X^{s s}$, i.e. que $X^{s s}$ a un quotient géométrique $M$, alors

$$
H_{G}^{*}\left(X^{s s}\right) \cong H^{*}(M),
$$

et, puisque $M$ est complet et n'a que des singularités quotient, $H^{*}(M)$ est pur, donc on a

$$
P_{p u r}^{G}\left(X^{s s}\right)=P(M)
$$

Il en résulte la formule de $F$. Kirwan

$$
P(M)=P(X) P\left(B_{G}\right)-\sum(-1)^{q} l^{2 d(\underline{\beta}, m)} P\left(Z_{\underline{\beta}, m}\right) P\left(B_{s t a b \underline{\beta}}\right),
$$

et comme corollaire que la décomposition $X=\cup S_{\beta}$ est équivariantement parfaite. 
Il faut souligner, toulefois, que F. Kirwan a déjà pronvé dans [15] que la stratification $X=\cup S_{\beta}$ est équivariantement parfaite mème diuns le cas général, et que ceci est équivalent à la pureté de $H_{C}^{*}\left(X^{s s}\right)$, mais nous n'avons pas de preuve directe de ce fait.

Exemple 2. Pour terminer ce paragraphe, nous donnons un dernier exemple d'application du corollaire an calcul des nombres de Betti des quotients géométriques étudiés par Bialynicki-Birula el Sommese ([5]). La situation considérée par ces auteurs est la suivante. Soient $T$ le tore $G_{m}, X$ un $T$-schéma lisse et complet sur $C$, et $U$ un ouvert $T$-invariant, de $X-X^{T}$ tel qu'il existe le quotient géométrique $U / T$ ' et est complet. Alors, il est prouvé par Bialynicki-Birula et Sommese que, si

$$
X=\bigcup_{\alpha \in A} X_{\alpha}^{+}=\bigcup_{\alpha \in A} X_{\alpha}^{-}
$$

sont les décompositions de $X$, il existe un sous-ensemble de $A$ non trivial $A^{-}$, tel que

$$
U=\bigcup_{\alpha \in A^{-}} X_{\alpha}^{+}-\bigcup_{\alpha \in A^{-}} X_{\alpha}^{-} .
$$

Nous allons retrouver les formules pour les nombres de Belti de $U / T$ obtenues dans $\{5\}$, en utilisant le corollaire à la proposition 6 . En effet, puisque

$$
\bigcup_{\alpha \in A^{-}} X_{\alpha}^{+}=U \cup \bigcup_{\alpha \in A^{-}} X_{\alpha}^{-}
$$

on a

$$
\sum t^{2 d_{\sigma}^{-}} P_{p u r}^{T}\left(X_{\alpha}^{+}\right)=P_{p u T}^{T}(U)+\sum t^{2 d_{\alpha}^{+}} P_{p u r}^{T}\left(X_{\alpha}^{-}\right)
$$

car rappelons que

$$
d_{\alpha}^{-}=\operatorname{din} X-\operatorname{dim} X_{\alpha}^{+}
$$

et

$$
d_{\alpha}^{+}=\operatorname{dim} X-\operatorname{dim} X_{\alpha}^{-} .
$$

On obtient donc

$$
P_{p u r}^{T}(U)=\sum t^{2 d_{\alpha}^{-}} P_{p u r}^{T}\left(X_{\alpha}^{+}\right)-\sum t^{2 d_{\alpha}^{+}} P_{p u r}^{T}\left(X_{\alpha}^{-}\right) .
$$

Or

$$
\begin{aligned}
P_{p+t r}^{T}\left(X_{\alpha}^{+}\right) & =P_{p a r r}^{T}\left(X_{\alpha}^{T}\right) \\
& =P\left(X_{\alpha}^{T}\right) P\left(B_{T}\right) \\
& =\frac{P\left(X_{\alpha}^{T}\right)}{1-t^{2}}
\end{aligned}
$$


et analogument

$$
P_{p n t}^{T}\left(X_{a}^{-}\right)=\frac{P\left(X_{a}^{T}\right)}{1-t^{2}}
$$

Et puisque

$$
P_{p a r}^{T}(U)=P(U / T)
$$

$U / T$ ćtant complet et à sirtgularités quotient, on obtient finalement

$$
P\left(U / T^{T}\right)=\sum_{a \in A^{-}} P\left(X_{a}^{T}\right) \frac{t^{20 t_{o s}^{-}}-t^{2} t_{t^{+}}^{+}}{1-t^{2}} .
$$

\section{Une version motivique}

Dans les paragraphes précédents, nous n'avons considéré que la filtration par le poids de la cohomologie, ar nous avons supposé $k$ de caractéristique quelconque; or si $k$ est un corps de caractéristique zéro, on peut formuler les résultats obtenus pour tout le système de réalisations de sir cohomologie ([10]) qui, en particulier; contient la structure de Hodge de cette cohomologié. Cette version motivique des résultats antérieurs s'obtient de la manière suivante.

Dans ce qui suit, on considlère donc $k$ un corps de caractéristique zéro. Soit $\operatorname{SRM}(k)$ la catégorie des systèmes de réalisations mixtes sur $k$, qui est une catégorie tanakienne ([10], cf. [13]), et soit $\operatorname{SRP}(k)$ la sous-catégoric tanakienne de $\mathbf{S R M}(k)$ engendrée par les systèmes de réalisations pures. Pour tout $m$, on a des foncteurs exacts

$$
G r_{m}^{W}: \operatorname{SRM}(k) \rightarrow \operatorname{SRP}(k)
$$

Nous noterons $K_{0} M(k)$ et $K_{0} P(k)$ le groupe $K_{0}$ de la catégorie abéliene $\operatorname{SRM}(k)$ et $\operatorname{SRP}(k)$, respectivenent. Ces groupes sont, de fait; isomorphes, car le morphisme

$$
\oplus_{m} G r_{m}^{w}: K_{0} M(k) \rightarrow K_{0} P(k)
$$

est un inverse du morplisme $K_{0} P(k) \rightarrow K_{0} M(k)$ induit par l'inclusion.

Si $X$ est un $k$-schéma, notons $\operatorname{SRM}^{*}(X)$ et $S R M_{c}^{*}(X)$ les systèmes de réalisations mixtes de $H^{*}(X)$ et $H_{c}^{*}(X)$, respectiventent $([10],[13])$.

On définit, dans $K_{0} M(k)$, les caractéristiques d'Euler motiviques de $X$ par

$$
\chi_{m o t}(X)-\sum_{i}(-1)^{i}\left[\operatorname{SRM}^{i}(X)\right]
$$


et

$$
\chi_{\text {mot }, c}(X)=\sum_{i}(-1)^{i}\left[\operatorname{SRM}_{c}^{i}(X)\right]
$$

Finalement, on définit, dans $K_{0} P(k)$, les caractéristiques d'Euler motiviques pures de $X$ par

$$
\chi_{m o t}^{m}(X)=\sum_{i}(-1)^{i} G r_{m}^{W}\left[\operatorname{SRM}^{i}(X)\right]
$$

et

$$
\chi_{m o t, c}^{m n}(X)=\sum_{i}(-1)^{i} G r_{m}^{W}\left[S R M_{c}^{i}(X)\right] .
$$

Après ces préliminaires, les versions motiviques des résultats des paragraphes précédents sont claires, par exemple la même preuve du théorème 1 mais avec $\chi_{m o t, c}^{m}$ et $S R M_{\mathrm{c}}^{i}$ au lieu de $\chi_{c}^{7 n}$ et $H_{c}^{i}$ nous donne:

Proposition 8. Soit $X$ un k-schéma et soit $X=\cup X_{\alpha}$ une décomposition de $X$. Alors on a

$$
\chi_{m o t, c}^{m}(X)=\sum_{\alpha} \chi_{\text {mot,c }}^{m}\left(X_{\alpha}\right) ; \quad \text { poutr tout } m
$$

Pour exprimer lą dualité de Poincaré dans ce contexte rappelons qu'on a dans $\operatorname{SRP}(k)$ des foncteurs exacts: lo foncteur de dualisation

$$
\begin{aligned}
\operatorname{SRP}(k) & \rightarrow \operatorname{SRP}(k) \\
M & \mapsto M^{\vee}:=\operatorname{Hom}(M, 1)
\end{aligned}
$$

et les twist de Tate

$$
\begin{aligned}
\operatorname{SRP}(k) & \rightarrow \operatorname{SRP}(k) \\
M & \mapsto M(n):=M \otimes \mathbf{1}(n) .
\end{aligned}
$$

Ces foncteurs induisent des morphismes

$$
\begin{aligned}
K_{0} P(k) & \rightarrow K_{0} P(k) \\
M & \mapsto M^{\vee}
\end{aligned}
$$

et

$$
\begin{aligned}
K_{0} P(k) & \rightarrow K_{0} P(k) \\
M & \mapsto M(n) .
\end{aligned}
$$

Alors, si $X$ est lisse, la dualité de Poincaré entraine

$$
\chi_{m o t, c}^{m}(X)=\left(\chi_{m o t}^{2 N-m}(X)\right)^{\vee}(-N),
$$

où $N=\operatorname{dim} X$, et on obtient immédiatement les résultats suivants. 
Corollaire. Si $X$ est lisse ef connere, et les cellules $X_{a}$ sont lisses, conneres et de codinension $d_{c k}$ dans $X$, on a

$$
\chi_{m o t}^{m}(X)=\sum_{a} \chi_{m o t}^{m-2 t o}\left(X_{0}\right)\left(-d_{c y}\right)
$$

Proposition 9. Soit $X$ un h-schéna connexe, lisse el complet sur lequal agit un tore alyábrique $T$. Si $X^{T}=\cup X_{c x}^{T}$ esi la décomposition de $X^{T}$ en composantes connexes el $d_{\mathrm{r}}$ est la codimension de la cellule $X_{6}$ de la décomposition de Buabrichi-Birula de $X$, on a

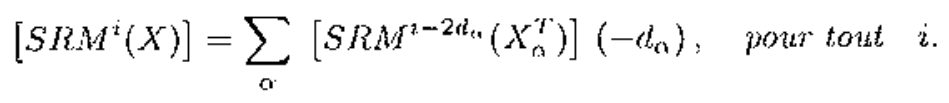

En particulier on en déduit l'égalité stumante entre nombres de Hodge (cf. $[6:)$

$$
h^{p P}(X)=\sum_{a} h^{p \cdots+t_{o r}, f_{t}-t_{\omega}}\left(X_{\alpha}^{T}\right)
$$

Finalement; nous établissons la version motivique du corollaire à la proposition 6, qui se démontre par les mêmes arguments que ce corollaire.

Proposition 10. Soit $X$ un G-schéma et soil $X=\cup X_{0}$ une $G$ décomposition de $X$. Si $X$ est tisse el connexe et les cellutes $X_{\alpha}$ sont lisses, equidimensionnelles et de codimension $d_{\Omega}$ dons $X$, on a

$$
P_{m o l, p u r}^{C r}(X)=\sum_{a} t^{2 d_{r r}} P_{m n o t, p u r}^{C G}\left(X_{G}\right)\left(-d_{c k}\right)
$$

Ainsi, pour l'exemple 2 du paragraphe 4 on obtient

$$
P_{m o l}(U / T)=\sum_{a \in A^{-}} P_{m a t}\left(X_{a}^{T}\right) \frac{t^{2 d_{c}^{-}}\left(-d_{c z}^{-}\right)-t^{2 t_{a}^{+}}\left(-d_{a}^{+}\right)}{1-t^{2}(-1)}
$$

d'où

$$
\left[S R M^{i}(U / T)\right]=\sum_{\alpha \in A^{-}} \sum_{d_{\alpha}^{-} \leq s<d_{c}^{+}}\left[S R M^{i-2 s}\left(X_{\alpha}^{T}\right)\right](-s)
$$

et, en particulier, on obtient pour les nombres de Hodge

$$
h^{p q}(U / T)=\sum_{a \in A^{-}} \sum_{d d_{2}^{-} \leq s<d_{\alpha}^{+}} h^{p-s, q-s}\left(X_{c 2}^{T}\right)
$$




\section{Bibliographie}

1. M. F. ATIYAH AND R. BOTT, The Yang-Mills equations over Riemann surfaces, Philos. Trans. Roy Soc. London Ser. A 308 (1982), $523-615$.

2. A. Bialynicki-BiRula, Some theorems on actions of algebraic groups, Ann. of Math. 98 (1973), 480-497.

3. A. BIALYNICKI-BirULA, On fixed points of torus actions on projective varieties, Bull. Acad. Polon. Sci. 22 (1974), 1097-1101.

4. A. Bialynicki-Birula, Some properties of the decompositions of algebraic varieties determinated by actions of a torus, Bull. Acad. Polon. Sci. 24 (1976), 667-674.

5. A. Bialynicki-BiRUla AND A. J. SOMmese, Quotiens by $\mathbb{C}^{*}$ and $S L(2, C)$ actions, Trans, Amer. Math. Soc. 279 (1983), 773-800.

6. J. B. Carrell and A. J. Sommese, Some topological aspects of $C^{*}$ actions on compact Kähler manifolds, Comment. Math. Helv. 54 (1979), 567-582.

7. P. DEIIGNE, Poids dans la cohomologie des variétés algébriques, in "Actes du Congres International des Mathematiciens," Vancouver, 1974 , pp. $79-85$.

8. P. Deiligne, Théorie de Hodge, II, Publ. I.H.E.S. 40 (1971), 5-58; III, Publ. L.H.E.S. 44 (1975), 5-77.

9. P. Deligne, La conjecture de Weil, II, Pub. I.H.E.S. 52 (1980), $137-252$.

10. P. DELiGNE, Le groupe fondamental de la droite projective moins trois points, in "Galois Groups over Q," Springer-Verlag, 1989, pp. 79-297.

11. A. DuRfeE, Euler characteristics in mixed Hodge theory, Preprint.

12. T. FRANKel, Fixed points and the torsion on Kähler manifolds, Ann. of Math. 70 (1959), 1-8.

13. U. JANNSEN, "Mixed Motives and Algebraic K-Theory,". Lectures Not. in Math. 1400, Springer-Verlag, 1990.

14. J. JuRkiewicz, An example of algebraic torus action wich determines the nonfiltrable decomposition, Bull. Acad. Polon. Sci. 25 (1977), 1089-1092.

15. F. KlRWAN, "Cohomology of quoliens in symplectic and algebraic geometry," Princeton Math. Notes 31, 1984. 
16. F. KiRWAN, Intersection homology and torus actions, Jour. Amer. Math. Soc. 1 (1988), 385-400.

17. H. Suminizo, Equivariant completions, J. Math. Kyoto Univ. 14 (1974), I,-28.

Departament d'Àlgebra i Geometria

Facultat de Matemàtiques

Universitat de Barcelona

Gran Via 585

E-08007 Barcciona

SPAIN

Rebut el 17 de Gener de 1992 